\title{
Study and Development of Temperature and Humidity Controller for Spreat Culti
}

\author{
Khongdet Phasinam ${ }^{1}$, Thanwamas Kassanuk ${ }^{2}$ \\ ${ }^{1,2}$ School of Agricultural and Food Engineering, Faculty of Food and Agricultural Technology, Pibulsongkram Rajabhat \\ University, Thailand \\ phasinam@psru.ac.th, ${ }^{2}$ t.kassanuk@gmail.com
}

\begin{abstract}
Consumable sprouts are considered as very popular foods because of many benefits and nutrients. Since the sprouts can be easily prepared according to various recipes, the needs of consumers are increased. However, growing the sprouts in open areas is complicated and the sprouts must be closely cared. The production costs are high. Moreover, the factors of the growths of the sprouts (e.g. environment, water quantity, humidity, temperature, pest and climate) cannot be controlled. These affect growing the sprouts. The sprouts should not also be contaminated with germs and viruses from planting materials. Therefore, the purposes of this research study are to study and to develop the temperature and relative humidity control devices for growing the sprouts in order to automatically control the temperature and humidity inside the device. According to the experimental results, it was found that the appropriate temperatures were $26-30$ degree Celsius. The suitable humidities were 90 - 95\% RH. The device consisted of the seven components as follows: 1) the control system, 2) the temperature and relative humidity sensors, 3) the ultrasonic humidifier, 4) the water container, 5) the fan for increasing the humidity, 6) the fan for decreasing the humidity and 7) the sprouting shelves. By comparing the experimental results with that of general planting methods, the growing time was reduced for $37 \%$. The used water quantity was lowered for 50 times. The sprouts could grow equally. The temperatures and humidities could be controlled as required by the sprouts.
\end{abstract}

Index Terms

Automatic Control System, Sprout, Growth Factor, Smart Farming

Article Received: 10 August 2020, Revised: 25 October 2020, Accepted: 18 November 2020

\section{Introduction}

Toxin-free vegetables, organic vegetables or sprouts such as sunflower sprouts, water spinach sprouts, microgreens and bean sprouts are crops having short production periods and being able to be produced all year. The vegetables can be produced for household consumptions or commercial purposes that can be a part-time job, develop food security as well as reduce the poverty and the lack of nutrients in developing countries because of the quick, simple and economical production and the high amounts of nutrients [1]. To produce the toxin-free vegetables, organic vegetables and sprouts having high qualities and safety for consumers, chemicals or hormones should not be used for stimulating the developments or growths of the plants [2], [3]. Presently, there are various methods for growing toxin-free vegetables and sprouts. There are also complicated methods requiring long time periods to nurture the plants. Some methods use chemical substances in order to maintain the freshness of the vegetables. The substances are harmful to consumers. Therefore, additional methods are invented and developed in order to improve watering the plants. Most developments are small devices that facilitate growing the plants. For example, timers are used for watering the plants and microcontrollers are used for releasing wastewater and circulating water. Planting machines that releases wastewater significantly waste water. Moreover, the water circulation systems may make the plants rotten easily because the environmental factors of the developments and growths of the sprouts as well as the sprouts should be free from the diseases and parasites from planting materials [4]. Furthermore, the concept of "smart farms" utilizes electronic, computer and information technologies that develop and facilitate farming in the form called smart farm system that applies scientific and technological knowledge according to the new economic development model of Thailand 4.0. It focuses on developing the country's economy by providing the technologies for farming. That is, it transforms traditional farming into modern farming that emphasizes on management and technologies (i.e. smart farming) [5]. Therefore, the purposes of this study are to design and develop temperature and relative humidity controller for automatically growing sprouts with the appropriate temperatures and relative humidity in order to solve the mentioned problems, to apply them to all sizes of devices, and to develop commercial devices.

\section{Research Objectives}

The purposes of this research study are to study and to develop the temperature and relative humidity control devices for growing the sprouts in order to automatically control the temperature and humidity inside the device.

\section{Research Methods}

\section{A. Designing the Temperature and Relative Humidity Controller}

To design the temperature and relative humidity controller for growing the plants, the engineering programs were used for designing and creating the 3D model in order to present the simulated dimensions of the shapes and components of the controllers that can ensure that the controllers and 
components were assembled correctly and consistently with the tasks [6]. The prototype was 40 centimeters wide, 40 centimeters long, and 80 centimeters high. It had the shelves for growing the sprouts. Each shelf was 10 centimeters from each other. There were three shelves. There was the water filling slot under the prototype. It has two sliding doors made of the acrylic materials. The appearance of the prototype was emphasized. It can be furniture in order to meet the needs of modern people. It has light weight. It is portable. It was a closed system in order to stabilize the temperature and relative humidity, to solve the climate problems obstructing the growths of the plants and causing rotten plants and to prevent the diseases and parasites as shown in Fig. 1. There were seven components as follows: 1) control system, 2) temperature and relative humidity sensor, 3) ultrasonic humidifier, 4) water container, 5) fan for increasing the humidity, 6) fan for decreasing the humidity, and 7) shelves for growing the plants, respectively, as shown in Fig. 2 and Fig. 3.
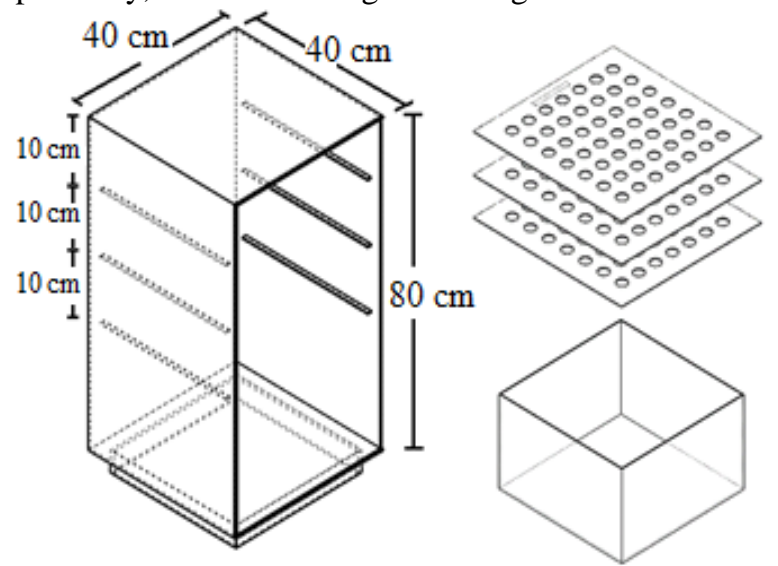

Fig. 1 The 3D model of the temperature and relative humidity controller

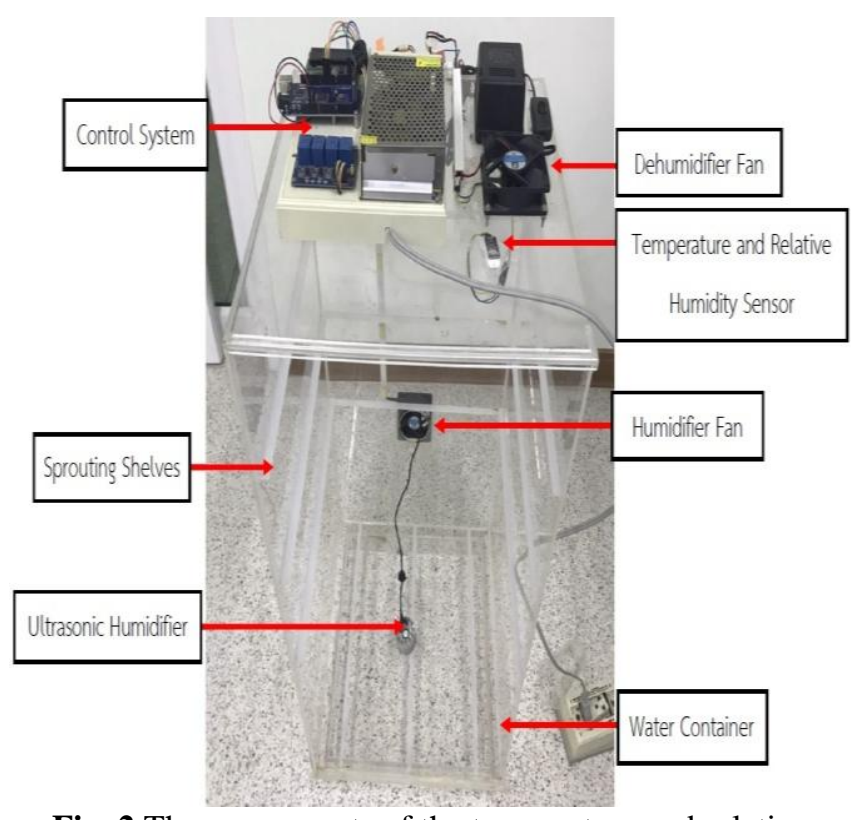

Fig. 2 The components of the temperature and relative humidity controller (in the front view)

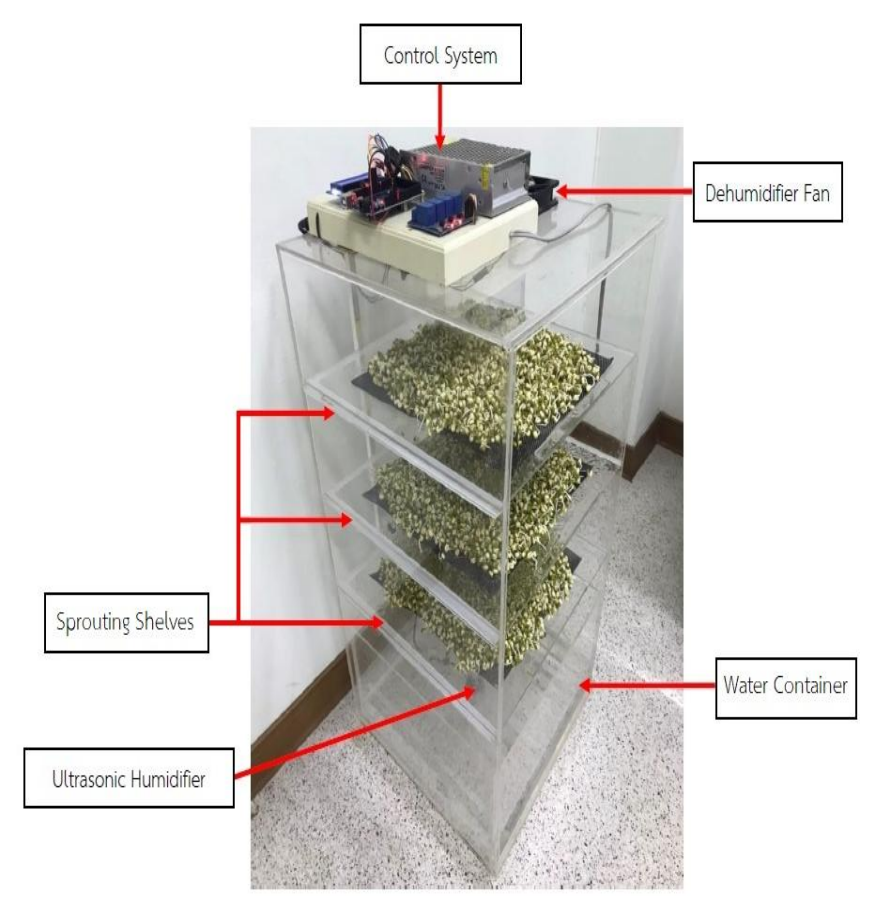

Fig. 3 The components of the temperature and relative humidity controller (in the isometric view)

\section{B. Deigning the Temperature and Relative Humidity Controller System}

The system controlling the environmental factor of the developments and growths of the sprouts used the microcontroller as the processing unit. The temperature and relative humidity sensor was installed through the processing unit in order to command the humidifier to adjust the humidity as required by the sprouts in the closed containers. There were the processes as shown in Fig. 4. The controller system consisted of the five main parts as follows: 1) the $220 \mathrm{~V}$ to $24 \mathrm{~V}$ converter; 2) the Arduino MEGA 2560 controller board developed from ATmega 2560 with the 256 kilobytes of flash memory, eight kilobytes of RAM, $7-12$ $\mathrm{V}$ power supply, 5V system, 54 digital inputs/outputs, and 14 pins supporting PWM outputs, 16 analog input pins, four UARTS (hardware serial ports), 4 megahertz of operating frequency, USB connection, AC to DC adapter, reset button, and connections to shields for connecting to Arduino Duemilanove or Diecimila; 3) the 220 V 10 A relay switch; 4) the $16 \times 2$ LCD screen for displaying temperature and relative humidity; and 5) the ON/OFF switch as shown in Fig. 5. 


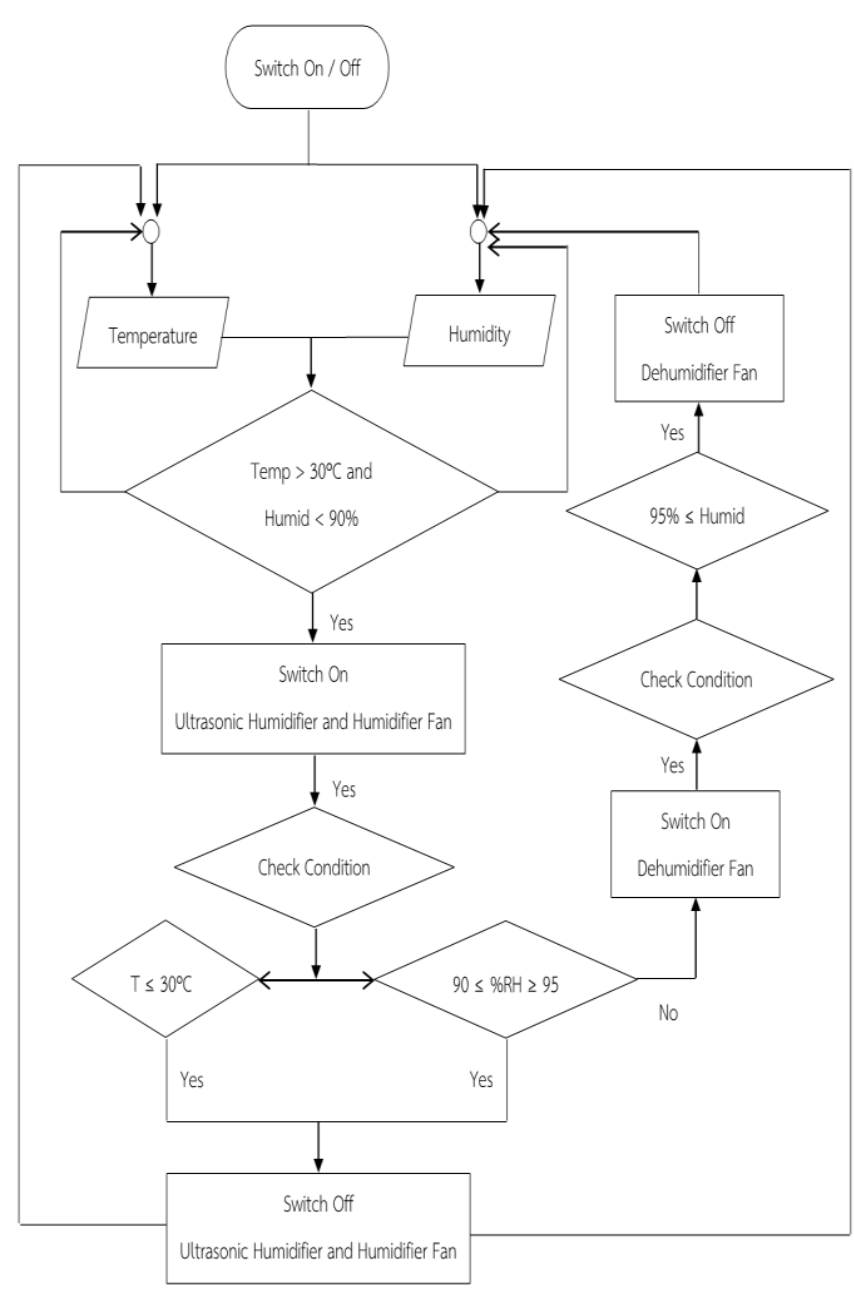

Fig. 4 Flow chart over all process

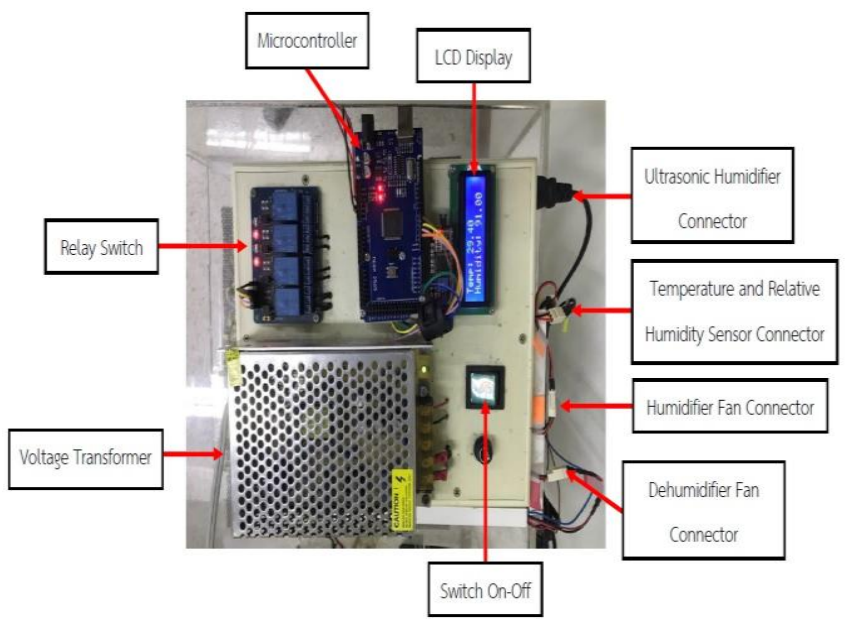

Fig. 5 The control system of the temperature and relative humidity controller

The temperature and relative humidity controller for growing the sprouts was a close system designed to be a package that can operate automatically. The controller had the DHT22 temperature and relative humidity sensor that had the high accuracy. It could measure the temperature within the range of -40 degree Celsius to 125 degree Celsius. Its deviation was \pm 0.5 degree Celsius. It could measure the relative humidity within the range of $0-100 \%$ $\mathrm{RH}$. The deviation was $\pm 2-5 \% \mathrm{RH}$. It had the $3-5 \mathrm{~V}$ power supply. It could measure the temperature and relative humidity inside the controller. It had the $24 \mathrm{~V}$ air ventilation fan reducing the humidity inside the controller if the relative humidity exceeds the specified limit, and it stopped working if the relative humidity reached the limit. The $24 \mathrm{~V}$ ultrasonic humidifier could create the fog inside the controller if the relative humidity was lower than the specified value, and the $24 \mathrm{~V}$ fan taking the air into the controller in order to increase the humidity created by the ultrasonic humidifier for each shelf until the relative humidity reaches the specified value as required by the sprouts. The ultrasonic humidifier and fan could automatically stop running. The control system received the data from the temperature and relative humidity inside the controller. If the relative humidity was lower than the specified value, then the system would send signals to the ultrasonic humidifier and fan to start running in order to increase the humidity of each shelf. If the relative humidity reached the specified value, then the control system would send signals to the ultrasonic humidifier and fan to instantly stop running. If the relative humidity was higher than the specified value, then the system would send signals to the fan to decrease the relative humidity in order to control the temperature and relative temperature as required by the sprouts.

\section{Testing the Temperature and Relative Humidity Controller for Growing the Sprouts}

The temperature and relative humidity controller for growing the sprouts was tested in order to check the operations of the controller by comparing the temperatures and relative humidity measured by the controller and that of the wet-dry bulb hygrometer. The data were collected every one hour for five days in order to compare the accuracies and errors. Then, it was tested by growing the sprouts in order to evaluate the results with the developed controller and to compare the results with that from the traditional method.

\section{Results}

By measuring the temperature and relative humidity with the temperature and relative humidity controller for growing the sprouts and comparing the temperature and relative humidity with that from the wet-dry bulb hygrometer with the data collected every hour for five days, it was found that the appropriate temperatures were 26 - 30 degree Celsius. The appropriate relative humidity was $90-95 \% \mathrm{RH}$. The percentage of errors of the temperatures measured by the controller was 5.00 percent, and that of the relative humidity measured by the controller was 2.97 percent as shown in Fig. 6 and Fig. 7. The sprouts grew during the whole harvesting period of 48 hours. The average length of the sprouts was $13.75 \mathrm{~cm}$ as shown in Fig. 8 . 
- Machine $\backsim$ Hygrometer $\llbracket \%$ Error

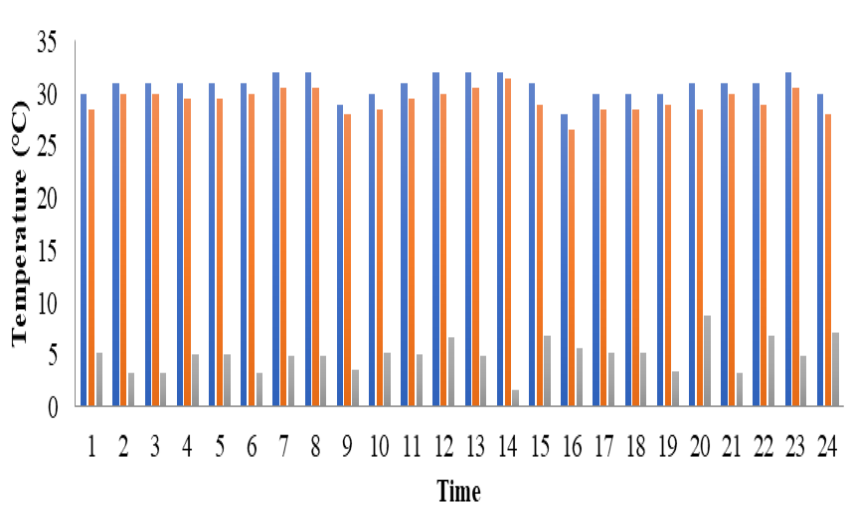

Fig. 6 The average temperature of the temperature and relative humidity controller and hygrometer for five days -Machine - Hygrometer $\quad \%$ Error

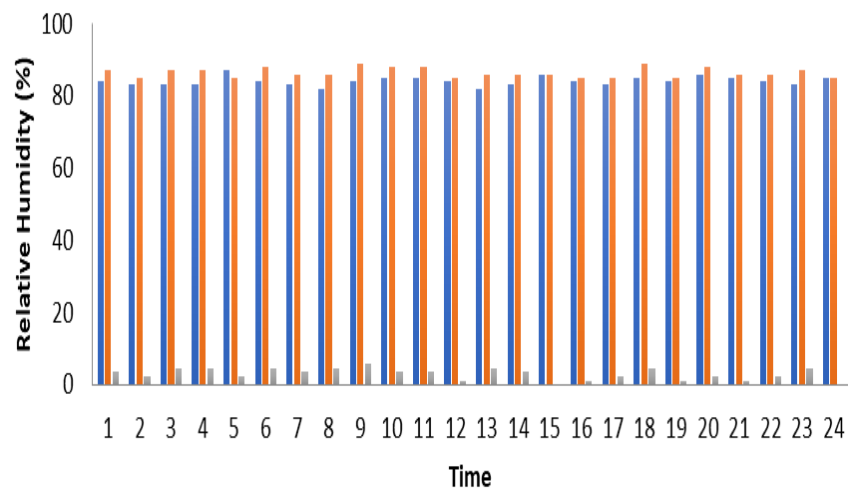

Fig. 7 The average relative humidity of the temperature and relative humidity controller and hygrometer for five days

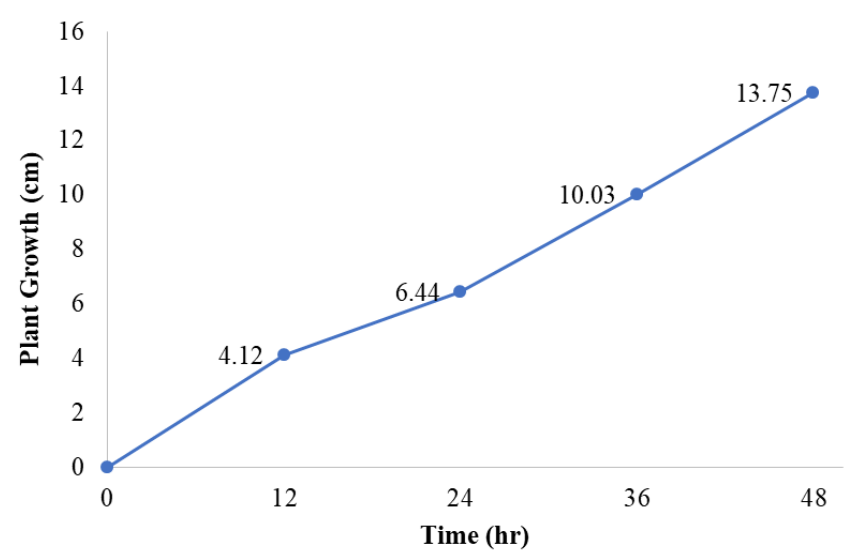

Fig. 8 The average growths of the sprouts during the whole harvesting period of 48 hours

\section{Discussions}

The present findings showed that the temperature and relative humidity affected the growths of the sprouts. According to the test results, it was found that the appropriate temperatures were $26-32$ degree Celsius. The appropriate relative humidity was $90-95 \% \mathrm{RH}$ [7]. This was consistent with S. Somwong and other [8], who developed the climate control system for growing microgreens. They also found that the appropriate temperature was lower than 30 degree Celsius as well as the appropriate relative humidity was $65-90 \%$, respectively. The average length of the vegetables was longer than that from the general methods for about 3.2 centimeters. The average weight of the vegetables was higher than that from the general methods for 70.51 percent. The harvesting period could be shortened for two to three days. For the developed temperature and relative humidity controller, the percentage of the errors about the temperature was $5 \%$. The percentage of the errors about the relative humidity was $2.97 \%$. This was acceptable. By comparing the results to that of the general methods, the growing period was shortened for $37 \%$. The water amount was reduced for 50 times. The sprouts could grow similarly.

\section{Recommendations}

\section{A. Recommendations for Practices}

1. The ultrasonic humidifier could reduce costs, especially for the water supply cost. It is suggested to be used by farmers or people interested in it.

2. The findings can reduce the costs regarding human resources and time. Particularly, the findings can be applied to plants with high values.

\section{B. Recommendations for Further Research}

1. Further studies applying this system to other plants should be conducted in order to obtain useful data.

2. This system used affordable measuring devices and control system as compared to the sensors in the market. The systems in the market are expensive, and the systems' climate settings cannot be set by users. Hence, the durability of devices should be tested in long terms.

\section{Conclusion}

The temperature and relative humidity controller for growing the sprouts could automatically control the temperature and relative humidity as required by the sprouts. The average percentage of the errors about the temperature and relative humidity was not higher than $5 \%$. This was acceptable. The harvesting period was shortened for $37 \%$. The water amount was reduced for 50 times. The sprouts could grow similarly, and the sprouts were toxin-free.

\section{Acknowledgement}

This is grateful for to Faculty of Food and Agricultural Technology, Pibulsongkram Rajabhat University for the research fund number $4 / 2560$.

\section{References}

[1] K. Lertrat, "Crop Production Technology for 21st Century," Khon Kaen Agriculture Journal, vol. 40, pp. 1-8, 2012.

[2] Office of Knowledge Management and Development. (2017). Planting of Organic 
Sprouts. [Online]. Available: http://www.okmd.or.th/knowledge-boxset/articles/farming/821/Planting-ofOrganic-Sprouts

[3] M. Hanif, M. K. Khattak, I. U. Haq, K. Gul, A. Khan, K. Ullah, A. Khan, and A. Ali, "Effects of Temperature and Water Purity on Germination and Yield of Mungbean Sprouts," Sains Malaysiana, vol. 48, pp. $711-717,2019$.

[4] I. A. Lakhiar, J. Gao, T. N. Syed, F. A. Chandio, and N. A. Buttar, "Modern Plant Cultivation Technologies in Agriculture Under Controlled Environment: A Review on Aeroponics," Journal of Plant Interactions, vol. 13, pp. 338 - 352, 2018.

[5] T. Pothong, P. Mekarun, and S. Choosumrong, "Development of Smart Farming Service System for Smart Farmer using FOSS4G and IoT," Naresuan Agriculture Journal, vol. 16, pp. $10-17$, 2019.

[6] T. Kassanuk1 and K. Phasinam, "Design and Construction of Water Hyacinth Chopper," RMUTI Journal Science and Technology, vol. 13, pp. 57 - 68, 2020.

[7] N. Hailu, C. Fininsa, T. Tana, and G. Mamo, "Effects of Temperature and Moisture on Growth of Common Bean and Its Resistance Reaction against Common Bacterial Blight (Xanthomonas axonopodis pv. phaseoli strains)," Journal of Plant Pathology and Microbiology, vol. 8, pp. $1-6,2017$.

[8] S. Somwong and M. Chongcheawchamnan, "Development of an Environmental Control System for Growing Microgreens," FEAT Journal, vol. 5, pp. $15-25,2019$. 